\title{
Assessment of optimal renin-angiotensin-system inhibition strategy in Asian patients with STEMI after primary myocardial revascularization
}

\author{
Hye-Jin Park ${ }^{1, \dagger}$, Ho-Jun Jang ${ }^{2, \dagger}$, Tae-Hoon Kim ${ }^{3, *, \dagger}{ }^{4}$, Sung Woo Kwon ${ }^{4, *, \dagger,}$ \\ Sang-Don Park ${ }^{4}$, Min Gyu Kong ${ }^{5}$, Jon Suh ${ }^{5}$, Pyung Chun $\mathrm{Oh}^{6}$, Jeonggeun Moon ${ }^{6}$, \\ Kyounghoon Lee ${ }^{6}$, Woong Chol Kang ${ }^{6}$ \\ ${ }^{1}$ Division of Infectious Diseases, Asan Medical Center, University of Ulsan College of Medicine, 05505 Seoul, Republic of Korea \\ ${ }^{2}$ Division of Cardiology, Sejong General Hospital, 21080 Bucheon, Republic of Korea \\ ${ }^{3}$ Division of Cardiology, CHA Ilsan Medical Center, CHA University School of Medicine, 10414 Goyang, Republic of Korea \\ ${ }^{4}$ Division of Cardiology, Inha University Hospital, 22332 Incheon, Republic of Korea \\ ${ }^{5}$ Division of Cardiology, Soon Chun Hyang University Bucheon Hospital, 14584 Bucheon, Republic of Korea \\ ${ }^{6}$ Cardiology Division, Department of Internal Medicine, Gil Medical Center, Gachon University College of Medicine, 21564 Incheon, Republic of Korea \\ *Correspondence: sch.kimtaehoon@gmail.com (Tae-Hoon Kim); kwonswdr@gmail.com (Sung Woo Kwon) \\ $\dagger^{\top}$ These authors contributed equally. \\ Academic Editor: Brian Tomlinson \\ Submitted: 3 June 2021 Revised: 4 August 2021 Accepted: 31 August 2021 Published: 14 January 2022
}

\begin{abstract}
Background: For the Asian patients with STEMI undergoing PCI, ACEIs are known to have a better outcome than ARBs. However, there is limited evidence to suggest so. Methods: Among the STEMI registry consist of 1142 Korean patients, we compared the MACE, the composite of myocardial infarction, stoke, death, admission for heart failure, and target vessel revascularization, between the ACEI and ARB groups (Set 1). Further, we defined adequate medication as the administration of a dose equal to or higher than the initiation dose of ACEI according to the heart failure guideline recommendation with a mandatory addition of beta-blockers, and compared the outcomes between the inadequate and adequate medication groups (Set 2). Propensity score matching was used to eliminate difference. Results: In the Set 1 comparison, patients in the ACEI group had a better outcome than those in the ARB group for both whole and matched populations (whole and matched population: Cox regression hazard ratio [HR], 0.645 and $0.535 ; 95 \%$ confidence interval [CI], $0.440-0.944$ and $0.296-0.967 ; p=0.024$ and $p=0.039$, respectively). In the Set 2 comparison for the whole population, patients in the inadequate medication group had more MACE than those in the adequate medication group (HR, $0.673 ; 95 \%$ CI, $0.459-0.985 ; p=$ 0.042). However, no difference was observed after propensity score matching (HR, $1.023 ; 95 \% \mathrm{CI}, 0.654-1.602 ; p=0.919)$. Conclusion: ACEIs might be a better choice than ARBs after primary revascularization. However, this study's findings suggest that early ACEI dose escalation combined with beta-blocker use may not improve prognosis.
\end{abstract}

Keywords: Myocardial infarction; Renin-angiotensin-aldosterone system; ACE inhibitors; Angiotensin receptor blocker; STEMI; Bradykinin

\section{Introduction}

The clinical benefit of angiotensin-converting enzyme inhibitors (ACEIs) in patients with myocardial infarction (MI) is well-known [1]. Angiotensin receptor blockers (ARBs) were introduced after ACEI, have a similar pathway of blocking the renin-angiotensin-aldosterone system (RAAS), and are permitted alternatives for use in patients with MI. Their role in MI has been studied in large-scale randomized controlled trials (RCTs) [2,3]. A clinical study reported findings that support the comparative advantage of ARB in MI [4]. However, whether ARB can substitute ACEI post MI is still a matter of debate. ACEI theoretically increases bradykinin level by inhibiting degradation, which improves endothelial function and control of the hypercoagulable state through the acute release of tissue plasminogen activator in post-MI patients [5]. Some clinical studies on Asian populations support the superiority of ACEI over
ARB in the clinical outcome patients, both with and without ST-segment elevation MI (STEMI) [6,7].

In the present study, we compared the outcomes following administration of ACEI with those following administration of ARB in STEMI patients who underwent primary revascularization therapy (PCI). ACEI is either prescribed without a beta-blocker, or a very small dose of a beta-blocker is introduced at the time of discharge due to a concern for a drop in blood pressure or cardiac output. This may decrease the benefits of the ACEI. Therefore, we additionally compared the outcomes between the group with an inadequate discharge dose of ACEI or use of ACEI without a beta-blocker and the group with proper use of ACEI with a beta-blocker. 


\section{Methods}

\subsection{Study population, definitions, and design}

Data from 1485 patients enrolled in the INTERSTELAR registry cohort between 2007 and 2015 were reviewed. The INTERSTELLAR registry was designed to find out the prognosis of the patients after primary revascularization for STEMI in four regional hospitals (ClinicalTrials.gov identifier: NCT02804958) in Korea. Most of the participants in this study were Koreans, while approximately 3\% were Chinese, Southeast Asian, Uzbekistani, and Mongolian. To compare the outcomes of separate strategies of RAAS blockade, we split the patients into two groups by two different standards. We excluded 58 patients with in-hospital death and 285 patients who were discharged without a RAAS blocking agent. Finally, 1142 patients were included in the first review.

Acute STEMI was diagnosed based on clinical information, including blood samples, chest pain, and 12-lead electrocardiography. An emergency primary revascularization call was made by $\geq 2$ attending cardiologists at the time of patient presentation. Exclusion criteria were previously diagnosed coronary artery disease, valvulopathy (more than moderate), cardiomyopathy, pericardial disease, or congenital heart disease.

The 2016 European Society of Cardiology (ESC) Guidelines for the diagnosis and treatment of acute and chronic heart failure recommend ACEIs as class I drugs in patients with asymptomatic left ventricular (LV) systolic dysfunction and a history of MI to prevent or delay the onset of heart failure (HF) and prolong life [8]. However, in the 2017 ESC Guidelines for STEMI, the treatment indications for ACEIs have been broadened, and ACEIs should be considered in all patients with STEMI [9]. According to the guidelines, we defined adequate medication as the use of a dose equal to or higher than the recommended initiation dose of ACEIs with a concomitant use of beta-blockers. In contrast, we defined the following RAAS inhibition strategies as inadequate: (1) any use of ARB; (2) discharge with a lower-than-recommended ACEI initiation dose; and (3) use of an ACEI without a beta-blocker. Evidence-based recommended initiation doses are: captopril $6.25 \mathrm{mg}$ thrice daily, enalapril (2.5 mg) twice daily, lisinopril ( $2.5 \mathrm{mg})$, and ramipril $(2.5 \mathrm{mg})$ daily.

The primary endpoint was major adverse cerebrovascular and cardiovascular events (MACE), defined as a composite of MI, stoke, all-cause death, readmission due to symptoms of HF after PCI, and ischemia-driven target vessel revascularization. If the patients had multiple events, the first clinical event only was included in this analysis. Medical chart reviews and/or standardize phone call were included in this study. Medical status of all patients (RAAS blockade and beta-blockers) was assessed at discharge as well as at the first outpatient visit.

For set 1 comparison, we divided the patient population according to the type of RAAS blocking agent re- gardless of beta-blocker use: ACEI versus ARB. Among 1142 patients, 784 were prescribed an ACEI and 358 were prescribed an $\mathrm{ARB}$ at discharge. In set 2, we compared the adequate ACEI medication group with an inadequate RAAS blockade medication group. Among 1142 patients discharged with a RAAS blocking agent, data from 1064 patients were available for review. The study protocol was received from the institutional review board of Sejong General Hospital (SGH 2019-08-010). Written informed consent was obtained from all patients, and we complied with the Declaration of Helsinki (6th revision).

\subsection{Data analysis and statistical methods}

Patient characteristics were compared between the two groups using a two-sample $t$-test or Mann-Whitney U test for continuous variables and Pearson's chi-square or Fisher's exact tests for categorical variables. Continuous data are expressed as the mean \pm standard deviation. Categorical variables are expressed as the number of objects and percentage. A Kaplan-Meier curve of MACE was created for each age group. The relationship between the time to MACE and use of ACEI and ARB was determined using Cox regression analysis with unadjusted and adjusted hazard ratios (HR).

For predisposition and to adjust the selection bias of RAAS inhibitor strategy, propensity score matching was used. The nearest neighbor method was used for the propensity matching algorithm, and 0.1 standard deviation was used for the caliper width with a matching ratio of $1: 1$. Co-variates for matching were chosen among those with $p$ $<0.10$ in Table 1 . To check the intergroup balance after matching, $p$ value and the absolute standardized difference were used. The Matchit package in R software was used for the propensity score matching. Survival analysis was performed using the package suvminer in R (version 3.4.4; The corresponding software code for the open-source $\mathrm{R}$ project for statistical computing is provided; www.r-project.org) and Statistical Package for the Social Sciences (SPSS 18.0; SPSS Inc., Chicago, IL, USA), and $p$ values $<0.05$ were considered statistically significant.

\section{Results}

From 2007 through 2015, 1142 patients out of 1485 survived and were discharged with an ACEI or ARB. "Mean duration of hospital stay was $5.8 \pm 23.0$ days". In set 1, 784 patients with ACEIs and 358 patients with ARBs were compared. In set 2, 412 patients with inadequate and 652 patients with adequate RAAS inhibition were compared. Table 1 shows the baseline clinical, echocardiographic, and angiographic characteristics of the study population. 
Table 1. Baseline clinical, echocardiographic and angiographic characteristics of the whole population in the 2 groups in each set.

\begin{tabular}{|c|c|c|c|c|c|c|}
\hline \multirow{2}{*}{ Variables (n) } & \multicolumn{3}{|c|}{ Set 1} & \multicolumn{3}{|c|}{ Set 2} \\
\hline & ARB (359) & ACEIs (784) & $p$-value & Inadequate (412) & Adequate (652) & $p$-value \\
\hline Age, years & $60.7 \pm 13.4$ & $59.2 \pm 12.8$ & 0.065 & $61.2 \pm 13.9$ & $58.7 \pm 12.5$ & 0.003 \\
\hline Systolic BP (mmHg) & $128.5 \pm 29.2$ & $125.7 \pm 26.6$ & 0.121 & $126.0 \pm 28.2$ & $127.1 \pm 26.7$ & 0.528 \\
\hline Heart rate (bpm) & $77.9 \pm 21.0$ & $76.7 \pm 18.4$ & 0.337 & $76.5 \pm 20.9$ & $77.2 \pm 18.2$ & 0.542 \\
\hline BMI $\left(\mathrm{kg} / \mathrm{m}^{2}\right)$ & $24.0 \pm 2.9$ & $24.2 \pm 3.2$ & 0.189 & $24.0 \pm 3.0$ & $24.3 \pm 3.2$ & 0.125 \\
\hline Males, n (\%) & $285(79.4)$ & $629(80.2)$ & 0.751 & $327(79.4)$ & $523(80.2)$ & 0.737 \\
\hline Hypertension, n (\%) & $202(56.3)$ & $338(43.1)$ & $<0.001$ & $205(49.8)$ & $290(44.5)$ & 0.093 \\
\hline DM, n (\%) & $110(30.6)$ & $181(23.1)$ & 0.007 & $131(31.8)$ & $146(22.4)$ & 0.001 \\
\hline Ever smoker, n (\%) & $193(53.8)$ & $365(46.6)$ & 0.024 & $262(63.6)$ & $289(44.3)$ & $<0.001$ \\
\hline Hypercholesterolemia & $112(31.2)$ & $104(13.3)$ & $<0.001$ & $111(26.9)$ & $89(13.7)$ & $<0.001$ \\
\hline eGFR & $69.1 \pm 26.4$ & $78.0 \pm 31.0$ & $<0.001$ & $71.2 \pm 29.4$ & $78.8 \pm 30.4$ & $<0.001$ \\
\hline CKD $>$ grade 3 & $117(32.7)$ & $214(27.3)$ & 0.068 & $135(32.8)$ & $170(26.1)$ & 0.018 \\
\hline Killip class & & & 0.005 & & & 0.005 \\
\hline $1-2$ & $308(86.0)$ & $707(91.5)$ & & $354(86.6)$ & $591(91.9)$ & \\
\hline $3-4$ & $50(14.0)$ & $66(8.5)$ & & $55(13.4)$ & $52(8.1)$ & \\
\hline \multicolumn{7}{|l|}{ Echocardiography } \\
\hline LVEDD & $49.3 \pm 4.7$ & $49.8 \pm 5.2$ & 0.135 & $49.8 \pm 4.8$ & $49.7 \pm 5.2$ & 0.935 \\
\hline E/e' & $12.5 \pm 5.1$ & $12.8 \pm 7.5$ & 0.527 & $12.6 \pm 5.0$ & $12.8 \pm 7.9$ & 0.704 \\
\hline $\mathrm{EF}$ & $48.0 \pm 9.6$ & $49.5 \pm 12.0$ & 0.028 & $51.3 \pm 10.5$ & $52.4 \pm 11.4$ & 0.222 \\
\hline LV dysfunction & 66 (18.7) & $184(23.7)$ & 0.063 & $93(23.0)$ & $150(23.2)$ & 0.934 \\
\hline \multicolumn{7}{|l|}{ Coronary disease } \\
\hline $1 \mathrm{VD}$ & $156(43.5)$ & $311(39.7)$ & 0.227 & $167(40.5)$ & $263(40.3)$ & 0.949 \\
\hline $2 \mathrm{VD}$ & $117(32.6)$ & $247(31.5)$ & 0.715 & $130(31.6)$ & $206(31.6)$ & 0.989 \\
\hline $3 \mathrm{VD}$ & $85(23.7)$ & $219(27.9)$ & 0.131 & $113(27.4)$ & $178(27.3)$ & 0.964 \\
\hline \multicolumn{7}{|l|}{ IRA } \\
\hline $\mathrm{LM}$ & $2(0.6)$ & $6(0.8)$ & $>0.99$ & $1(0.2)$ & $6(0.9)$ & 0.259 \\
\hline LAD & $181(50.4)$ & $397(50.6)$ & 0.945 & $204(49.5)$ & $336(51.5)$ & 0.521 \\
\hline LCX & $32(8.9)$ & $86(11.0)$ & 0.346 & $34(8.3)$ & 73 (11.2) & 0.120 \\
\hline RCA & $143(39.8)$ & $288(36.7)$ & 0.316 & $171(41.5)$ & $232(35.6)$ & 0.052 \\
\hline \multicolumn{7}{|l|}{ Deployed stents } \\
\hline Zotarolimus & $96(26.7)$ & $192(24.5)$ & 0.416 & $124(30.1)$ & $157(24.1)$ & 0.030 \\
\hline Everlolimus & $134(37.3)$ & $393(50.1)$ & $<0.001$ & $191(46.4)$ & $316(48.5)$ & 0.503 \\
\hline Biolimus & $40(11.1)$ & $35(4.5)$ & $<0.001$ & $38(9.2)$ & $32(4.9)$ & 0.006 \\
\hline Others* & $38(10.6)$ & $68(8.7)$ & 0.323 & $12(2.9)$ & $63(9.7)$ & $<0.001$ \\
\hline Stent diameter, mm & $3.36 \pm 1.28$ & $3.36 \pm 0.49$ & 0.961 & $3.27 \pm 0.45$ & $3.37 \pm 0.50$ & 0.001 \\
\hline Stent length, mm & $26.0 \pm 10.6$ & $26.8 \pm 11.5$ & 0.334 & $26.5 \pm 10.4$ & $26.8 \pm 11.7$ & 0.702 \\
\hline \multicolumn{7}{|l|}{ Medications } \\
\hline Beta-blockers (\%) & $338 / 357(94.7)$ & $742 / 782(94.9)$ & 0.886 & & & \\
\hline Potent antiplatelet & $105(29.2)$ & $200(25.5)$ & 0.185 & $99(24.0)$ & $175(26.8)$ & 0.307 \\
\hline Tica or prasugrel & $61(17.0)$ & $33(4.2)$ & $<0.001$ & $57(13.8)$ & $30(4.6)$ & $<0.001$ \\
\hline Statin & $319(88.9)$ & $671(85.6)$ & 0.132 & $361(87.6)$ & 565 (86.7) & 0.648 \\
\hline
\end{tabular}

BP, blood pressure; ER, emergency room; BMI, body mass index; DM, diabetes mellitus; eGFR, estimated Glomerular Filtration Rate was calculated by the Cockcroft-Gault formula; CKD, chronic kidney disease; LVEDD, left ventricle end-diastolic dimension; E/e', the ratio of mitral peak velocity of early filling (E) to early diastolic mitral annular velocity (e'); EF, ejection fraction; LV dysfunction, defined as ejection fraction $<40 \% ; 1 \mathrm{VD}$, one-vessel coronary artery disease; $2 \mathrm{VD}$, two-vessel coronary artery disease; $3 \mathrm{VD}$, three-vessel coronary artery disease; IRA, infarction-related artery; LM, left main; LAD, left anterior descending; LCX, left circumflex artery; RCA, right coronary artery; *others, other second-generation stent, "potent antiplatelet; dual antiplatelet therapy plus cilostazol or dual antiplatelet therapy with ticagrelor/prasugrel; Tica, ticagrelor. 
3.1 Differences in participant characteristics In set 1 and set 2

In set 1 , patients in the ARB group were older (60.7 \pm 13.4 years vs. $59.2 \pm 12.8$ years, $p=0.065)$ and had a higher prevalence of hypertension $(56.3 \%$ vs. $43.1 \%, p$ $<0.001)$, diabetes $(30.6 \%$ vs. $23.1 \%, p=0.007)$, hypercholesterolemia $(31.2 \%$ vs. $13.3 \%, p<0.001)$ and eversmoking (53.8\% vs. $46.6 \%, p=0.024)$ compared to those in the ACEI group. The initial Killip class was higher in the ARB group (Killip class $3-4,14.0 \%$ vs. $8.5 \%, p=0.005$ ). Although the mean ejection fraction was lower (48.0 \pm $9.6 \%$ vs. $49.5 \pm 12.0 \%, p=0.028), \mathrm{LV}$ dysfunction was less common in the ARB group (18.7\% vs. $23.7 \%, p=0.063)$. The rate of single-vessel coronary disease was similar in the two groups $(43.5 \%$ vs. $39.7 \%, p=0.227)$ and the left anterior descending artery was the most common infarct-related artery in both groups $(50.4 \%$ vs. $50.6 \%, p=0.945)$. The stent diameters were also similar between the groups (3.36 $\pm 1.28 \mathrm{~mm}$ vs. $3.36 \pm 0.49 \mathrm{~mm}, p=0.961)$. Beta-blockers were prescribed to most patients, and no intergroup difference was noted $(94.7 \%$ vs. $94.9 \%, p=0.886)$. Newer potent antiplatelet therapy, including ticagrelor or prasugrel, was prescribed more frequently for patients in the ARB group $(17.0 \%$ vs. $4.2 \%, p<0.001)$.

A total of 1064 patients were enrolled for the set 2 comparison, with 412 and 652 patients assigned to the inadequate medication and adequate medication groups, respectively. Patients in the inadequate group had a higher age $(61.2 \pm 13.9$ vs. $58.7 \pm 12.5, p=0.003)$ and prevalence of diabetes $(31.8 \%$ vs. $22.4 \%, p=0.001)$, hypercholesterolemia $(26.9 \%$ vs. $13.7 \%, p<0.001)$, ever-smoking $(63.6 \%$ vs. $44.3 \%, p<0.001)$, and initial Killip class (Killip class $3-4,13.4 \%$ vs. $8.1 \%$; $p=0.005$ ).

The stent diameters were similar in the 2 groups ( 3.27 $\pm 0.45 \mathrm{~mm}$ vs. $3.37 \pm 0.50 \mathrm{~mm}, p=0.001)$. Newer potent antiplatelet therapy, including ticagrelor or prasugrel, was more commonly prescribed for patients in the inadequate group $(17.0 \%$ vs. $4.2 \%, p<0.001)$.

In this study, we analyzed physicians' ARB preference for patients with renal dysfunction. As shown in Table 1, eGFR was lower in the ARB group $(69.1 \pm 26.4$ vs. $78.0 \pm$ $31.0 ; p<0.001$ ), and there was a trend toward patients with chronic kidney disease (CKD) $>$ grade 3 to be included in the ARB group (32.7\% vs. $27.3 \% ; p=0.068)$. Similarly, the mean eGFR was lower $(71.2 \pm 29.4$ vs. $78.8 \pm 30.4 ; p$ $<0.001)$ and a higher number of patients with CKD $>$ grade 3 were included in the inadequate medication group $(32.8 \%$ vs. $26.1 \% ; p=0.018$ ).

\subsection{Propensity score-matched population}

In set 1, 278 pairs of patients were obtained after 1:1 propensity score matching. The parameters are listed in Table 2. Table 3 shows the comparison between the groups with adequate use of RAAS inhibitors and inadequate use of ACEI. There were no differences in age, body mass in- dex, ejection fraction, and prevalence of diabetes, dyslipidemia, ever-smoking, hypertension, $\mathrm{CKD}>$ grade 3 , multivessel coronary artery disease, use of ticagrelor and prasugrel, Killip class 3-4, and the left anterior descending artery as the culprit vessel between the groups. We categorized stent diameter in four groups as follows: (1) $2.5-3.0 \mathrm{~mm}$; (2) $3.25-4 \mathrm{~mm}$; (3) $\geq 4.5 \mathrm{~mm}$; and (4) $2.25 \mathrm{~mm}$. There was no intergroup difference in the beta-blockers prescription rate $(94.9 \%$ vs. $93.8 \%, p=0.712)$. There was no difference in the stent diameter between the groups. Likewise, there were no baseline differences between the continuous and categorical variables after matching for set 2 comparison (Supplementary Fig. 1. The figure shows the standardized mean differences before and after propensity matchings In set 1 and set 2 comparisons.)

Table 2. Matched variables after propensity matching in patients on ARB vs. ACEIs (set 1).

\begin{tabular}{|c|c|c|c|c|c|}
\hline \multirow{2}{*}{ Set 1} & \multicolumn{2}{|c|}{$\mathrm{ARB}(\mathrm{n}=278)$} & \multicolumn{2}{|c|}{ ACEIs $(n=278)$} & \multirow{2}{*}{$p$-value } \\
\hline & Mean & SD & Mean & SD & \\
\hline \multicolumn{6}{|l|}{ Continuous variables } \\
\hline Age (years) & 59.8 & 12.7 & 61.1 & 13.5 & 0.250 \\
\hline BMI $\left(\mathrm{kg} / \mathrm{m}^{2}\right)$ & 24.3 & 3.4 & 24.0 & 2.8 & 0.202 \\
\hline Ejection fraction & 49.0 & 12.9 & 48.9 & 9.6 & 0.900 \\
\hline Stent length (mm) & 27.1 & 12.6 & 25.8 & 10.4 & 0.202 \\
\hline Categorical variables & $\mathrm{n}$ & Percent & $\mathrm{n}$ & Percent & \\
\hline DM & 71 & 25.5 & 73 & 26.3 & 0.846 \\
\hline Dyslipidemia & 62 & 22.3 & 66 & 23.7 & 0.687 \\
\hline Ever smoker & 130 & 46.8 & 140 & 50.4 & 0.396 \\
\hline Hypertension & 139 & 50.0 & 146 & 52.5 & 0.552 \\
\hline CKD $>$ grade 3 & 90 & 32.4 & 86 & 30.9 & 0.784 \\
\hline MVD & 95 & 34.2 & 99 & 35.6 & 0.721 \\
\hline Tica or prasugrel & 25 & 9.0 & 18 & 6.5 & 0.266 \\
\hline Killip class 3 or 4 & 30 & 10.8 & 32 & 11.5 & 0.787 \\
\hline Culprit LAD & 129 & 46.4 & 137 & 49.3 & 0.497 \\
\hline \multicolumn{6}{|l|}{ Stent diameter (mm) } \\
\hline $2.5-3$ & 184 & 66.2 & 194 & 69.8 & 0.568 \\
\hline $3.25-4$ & 92 & 33.1 & 80 & 28.8 & \\
\hline $4.5-5$ & 2 & 0.7 & 3 & 1.1 & \\
\hline 2.25 & 0 & 0 & 1 & 0.4 & \\
\hline
\end{tabular}

BMI, body mass index; EF, ejection fraction; DM, diabetes mellitus; CKD, chronic kidney disease; MVD, multi-vessel coronary disease; Tica, ticagrelor; LAD, left anterior descending artery; stent diameter was divided four groups by diameters; SD, Standard deviation.

\subsection{Clinical outcome of whole and matched population}

In set 1 , the incidence of MACE in the whole population during the follow-up was lower in the ACEI group than in the ARB group (Cox regression HR, 0.645; 95\% 
Table 3. Matched variables after propensity matching in patients with inadequate use vs. adequate use of renin-angiotensin-aldosterone system inhibitors (set 2).

\begin{tabular}{|c|c|c|c|c|c|}
\hline \multirow{2}{*}{ Set 2} & \multicolumn{2}{|c|}{ Inadequate use $(\mathrm{n}=327)$} & \multicolumn{2}{|c|}{ Adequate use $(\mathrm{n}=327)$} & \multirow{2}{*}{$p$-value } \\
\hline & Mean & SD & Mean & SD & \\
\hline \multicolumn{6}{|l|}{ Continuous variables } \\
\hline Age & 60.9 & 13.6 & 59.9 & 12.6 & 0.322 \\
\hline $\operatorname{BMI}\left(\mathrm{kg} / \mathrm{m}^{2}\right)$ & 24.0 & 2.9 & 24.1 & 3.0 & 0.715 \\
\hline Ejection fraction (\%) & 48.1 & 10.4 & 48.6 & 11.5 & 0.547 \\
\hline Stent length (mm) & 26.4 & 10.3 & 26.6 & 10.6 & 0.875 \\
\hline Categorical variables & $\mathrm{n}$ & Percent & $\mathrm{n}$ & Percent & \\
\hline DM & 86 & 26.3 & 86 & 26.3 & $>0.999$ \\
\hline Dyslipidemia & 64 & 19.6 & 58 & 17.7 & 0.547 \\
\hline Ever smoker & 195 & 59.6 & 189 & 57.8 & 0.633 \\
\hline Hypertension & 151 & 46.2 & 144 & 44.0 & 0.582 \\
\hline CKD $>$ grade 3 & 98 & 30.0 & 96 & 29.4 & 0.932 \\
\hline MVD & 108 & 33.0 & 110 & 33.6 & 0.868 \\
\hline Tica or prasugrel & 20 & 6.1 & 25 & 7.7 & 0.439 \\
\hline Killip class 3 or 4 & 34 & 10.4 & 31 & 9.5 & 0.695 \\
\hline Culprit LAD & 164 & 50.2 & 170 & 52.0 & 0.638 \\
\hline \multicolumn{6}{|l|}{ Stent diameter $(\mathrm{mm})$} \\
\hline $2.5-3$ & 234 & 71.6 & 229 & 70.0 & 0.898 \\
\hline $3.25-4$ & 91 & 27.8 & 96 & 29.4 & \\
\hline $4.5-5$ & 2 & 0.6 & 2 & 0.6 & \\
\hline 2.25 & 0 & 0 & 0 & 0 & \\
\hline
\end{tabular}

BMI, body mass index; EF, ejection fraction; DM, diabetes mellitus; MVD, multi-vessel coronary disease; Tica, ticagrelor; LAD, left anterior descending artery; stent diameter was divided four groups by diameters; SD, Standard deviation.

confidence interval [CI], $0.440-0.944 ; p=0.024)$. The outcome was similar after propensity matching (Cox regression HR, 0.535; 95\% CI, 0.296-0.967; $p=0.039$ ). The Kaplan-Meier survival curves for MACE for the whole and propensity-matched population are shown in Fig. 1.

In set 2 , the incidence of MACE during follow-up was higher in the inadequate medication group than in the adequate medication group (HR, 0.673; 95\% CI, 0.459-0.985; $p=0.042$ ). The outcome did not differ between the two groups after propensity score matching (HR, 1.023; 95\% CI, 0.654-1.602; $p=0.919$ ) (Fig. 2).

\section{Discussion}

The first key finding of our study is that among discharged patients with STEMI, those on an ACEI have a better clinical outcome than those on an ARB. However, secondary findings suggest that a sufficient dose of ACEI with a mandatory combination of beta-blockers at discharge was not necessarily associated with a better prognosis.

After primary revascularization, the most important medical therapies other than administration of antiplatelets and statins are RAAS blockade and administration of betablockers. With the transition to the era of reperfusion, the importance of beta-blockers has diminished, while the importance of RAAS inhibition is increasing. Recently, with the development of new ARBs and reports of their better efficacy, there is a growing expectation that ARB treatment would result in a better prognosis than ACEI treatment for patients with STEMI. However, based on recently released real-world registry data, especially data from Asia, there is accumulating evidence that ACEI is associated with improved prognosis in patients with MI. Byun et al. [6], reported that ACEI use was associated with a two-year reduction in MACE and revascularization rates for NSTEMI and DM patients. Similarly, in the STEMI patient subset, ACEI plus beta-blocker use was related to a two-year decrease in MACE rate. Current research shows that ACEI treatment is associated with improvements in MACE over five years, similar to the previous two-year results [7].

\subsection{ACEI and $A R B$}

Although both ACEI and ARB act by blocking a similar pathway in RAAS, their mechanisms of action differ. Theoretically, the beneficial effects of ACEI are strongly associated with increased levels of bradykinin. Bradykinin acts as a potent vasodilator by activating endothelial $B_{2}$ 
A

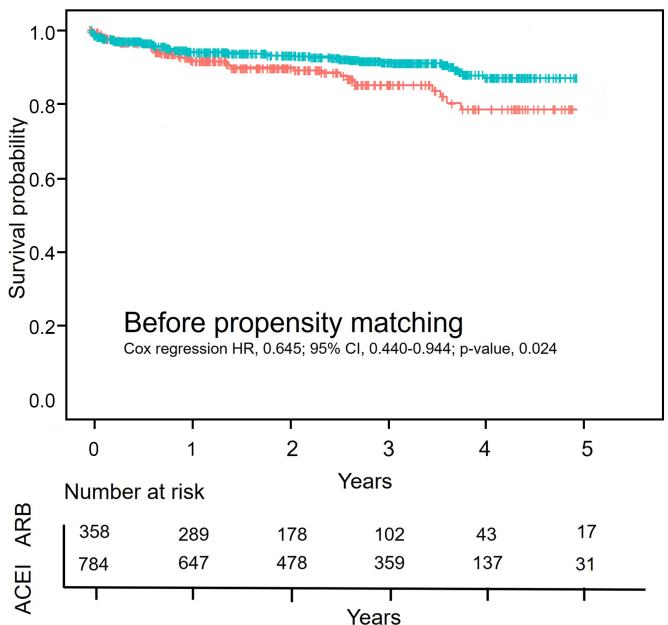

Red line: ARB

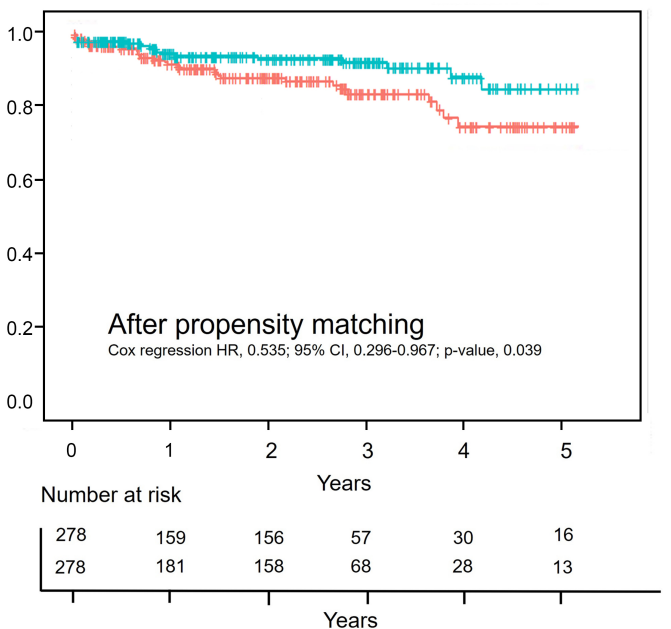

Fig. 1. The Kaplan-Meier survival curves for the composite of MACE (major adverse cardiac events; composite of myocardial infarction, stroke, all-cause death, readmission for heart failure, or target vessel revascularization) according to the use of angiotensin receptor blocker (ARB) or angiotensin-converting enzyme inhibitor (ACEI). (A) The left Kaplan-Meier curves shows comparison in the whole population. (B) The right Kaplan-Meier curves for the above medication groups in the matched population.

A Blue line: Adequate ACEl use group

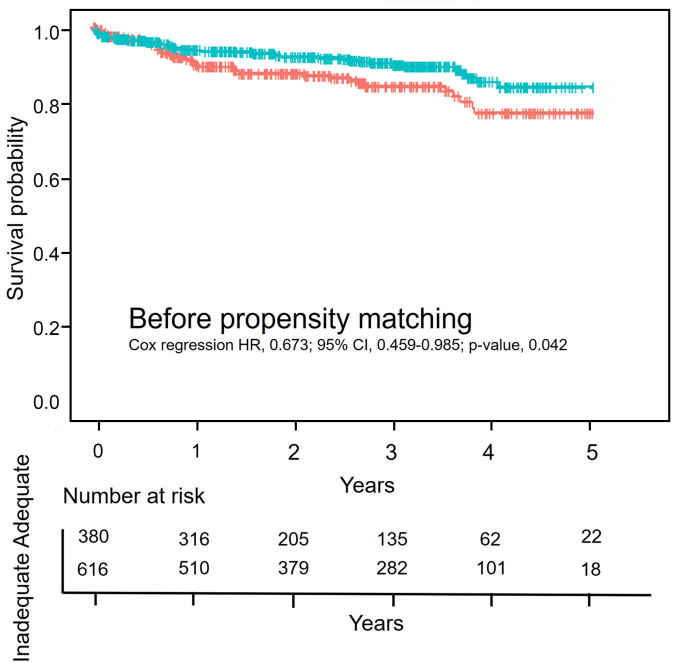

Red line: Inadequate RAAS use group

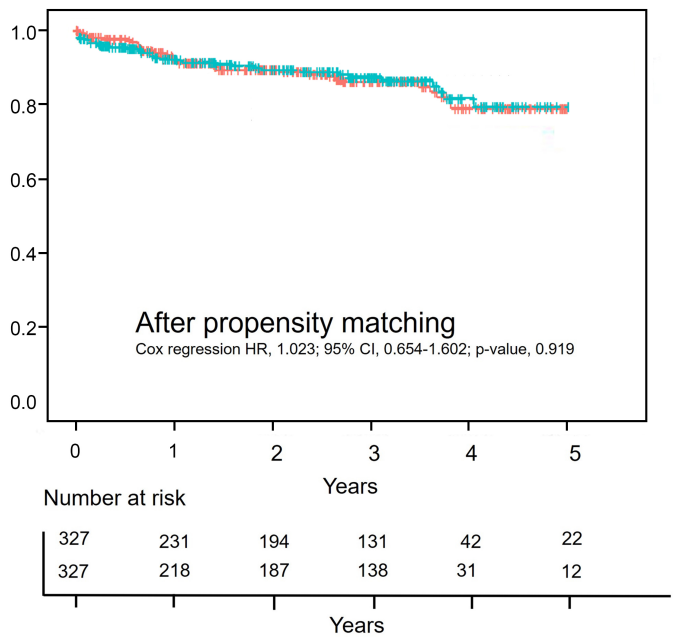

Fig. 2. The Kaplan-Meier survival curves for the composite of MACE (major adverse cardiac events; composite of myocardial infarction, stroke, all-cause death, readmission for heart failure, or target vessel revascularization) according to the inadequate use of renin-angiotensin-aldosterone system (RAAS) antagonist and its adequate use. An inadequate use of RAAS antagonist is defined as: any use of ARB; discharge with an initiation dose lower than the guideline recommendation; use of ACEI without a beta-blocker. (A) The left Kaplan-Meier curves shows comparison in the whole population. (B) The right Kaplan-Meier curves for the above medication groups in the matched population.

receptors. Prostacyclin, nitric oxide, and endotheliumderived hyperpolarizing factor are stimulated by its release $[10,11]$. The risk of acute MI was significantly lower with ACEI than that with placebo in large randomized trials. In the HOPE study, ramipril had a $20 \%$ lower event rate, and in the EUROPA study, perindopril had a $22 \%$ lower event rate $[12,13]$.

ARBs work by selectively blocking angiotensin II type 1 (AT1) receptors. The compensatory increase in angiotensin II levels may mediate harmful vascular effects via 
other angiotensin II receptors (AT2, 3, and 4), resulting in plaque instability or rupture $[14,15]$. The risk of acute MI was slightly higher with ARB (candesartan and irbesartan) than with placebo $[16,17]$.

Ferrari et al. [18,19] compared the vascular and endothelial functions between ACEIs and ARBs and found that ACEIs produce a greater reduction in endothelial dysfunction, inflammation, cell adhesion, and apoptosis, and have better antithrombotic and anti-atherosclerotic effects. ARB has only partial anti-thrombotic and inflammatory benefits. The ONTARGET study compared ACEI and $\mathrm{ARB}$ and showed a lower tendency for the risk of MI with ramipril as compared to telmisartan (relative risk, 1.07; 95\% CI, 0.94-1.22).

\subsection{Dose of ACE inhibitor and mandatory combination of beta-blocker}

In addition to choosing between ACEI and ARB, the clinically difficult decision about hemodynamic drug prescription would be which medicine to start with, and at what dose. A combination strategy of prescribing RAAS antagonists with beta-blockers might be a clinical concern when the patient's blood pressure is low. Some physicians start with one type of hemodynamic drug and increase the dose quickly, while others start with two drugs (ACEI and betablocker) and gradually increase the dose. Interestingly, previous studies or guidelines do not conclusively favor one strategy over the other. Importantly, high-risk patients with LV dysfunction might require both classes of medicine before discharge; nevertheless, they usually have a higher chance of developing hemodynamic instability during hospital stay. Thus, we attempted to analyze set 2 for these clinical concerns.

Patients undergoing primary PCI usually stabilize after pain control. Some patients may experience hypotension or compensatory tachycardia after the procedure. The treatment strategies at this stage may differ depending on the physician's experience. We did not include patients who either died before discharge or did not receive RAAS inhibitors or beta-blockers due to instability (343/1485, 23.0\%). The 2017 ESC and 2013 American College of Cardiology/American Heart Association guidelines for STEMI recommend that ACEI should be considered in all patients with STEMI [20,21]. Unlike the recommendation that betablockers should be used before discharge, ACEI are recommended within the first $24 \mathrm{~h}$. According to the guidelines, a RAAS inhibitor should be considered first, followed by a beta-blocker. However, due to unstable vital parameters, a lower-than-recommended starting dose may be prescribed and not titrated until discharge. Similarly, unstable patients may not receive beta-blockers until discharge. There is evidence that, in practice, a small number of patients receive suboptimal doses of ACEI [22]. Interestingly, the use of a dose equal to or higher than the recommended initiation dose of ACEI according to the HF guideline with a manda- tory addition of beta-blockers was not necessarily associated with a good prognosis.

There are several reasons that may explain the observed results. First, the role of a beta-blocker is not as strong as that of an ACEI in the primary PCI era. Betablockers are known to reduce mortality in patients with acute MI; however, most of this evidence comes from the pre-reperfusion era [23]. We must be aware that hospitalization rates for HF may increase further with the use of beta-blockers in the reperfusion era [23]. Second, in the absence of HF or systolic dysfunction, an equal risk of death was observed, regardless of the use of beta-blockers [24]. Indeed, we had less than a quarter of the population with LV dysfunction (ejection fraction $<40 \%$ ) in our study. Third, the impact of the ACEI dose at discharge might not be as high as the use of ACEI itself. It is apparent that a higher dose of ACEI is more beneficial for patients with a reduced ejection fraction [25]. Fourth, the correlation between the higher dose of a RAAS inhibitor and better prognosis seems to become weaker in the reperfusion era [26].

\section{Limitation}

Our study had several limitations. First, the sample size was small. Second, the analysis was conducted using non-randomized registry data. However, we used propensity score matching to minimize the selection bias. Third, the use of a RAAS inhibitor was observed at discharge as well as the first outpatient visit. Thus, the delayed titration strategies were not assessed.

\section{Conclusions}

ACEIs might be a better choice than ARBs after primary PCI in patients with STEMI. However, this study's findings suggest that early ACEI dose escalation in combination with beta-blocker use may not improve prognosis. A well-designed prospective dose-related study of ACEIs versus ARBs in patients with MI is warranted to confirm our findings.

\section{Abbreviations}

STEMI, ST-elevation myocardial infarction; PCI, percutaneous coronary intervention; ACEI, angiotensinconverting enzyme inhibitor; ARB, angiotensin receptor blocker; RAAS, renin-angiotensin-aldosterone system; MACE, major adverse cardiovascular and cerebrovascular event; MI, myocardial infarction; RCT, randomized controlled trial; $\mathrm{CKD}$, chronic kidney disease; CI, confidence interval; HR, hazard ratio.

\section{Author contributions}

HJP, HJJ, and THK wrote the manuscript; JS, SWK, SDP, MGK and PCO collected and analyzed data; JM, KL, WCK, MGK and THK performed statistical analyses; THK, HJJ, and SWK conceived and designed the study. 


\section{Ethics approval and consent to participate}

The analysis was conducted using data obtained from the INTERSTELLAR registry of patients with STEMI who underwent primary PCI. The INTERSTELLAR registry (clinicaltrials.gov identifier NCT02804958; Am J Cardiol 2016 Jul 15;118(2):177-82; https://doi.org/10.1371/jo urnal.pone.0159416) is a retrospective, observational, fourregional-hospital-based registry that reflects management practices, risk factors, and clinical outcomes in patients with STEMI who underwent primary PCI in the cities of Incheon and Bucheon located in the mid-western part of the Korean peninsula between 2007 and 2014. All participants provided informed consent for inclusion before they participated in the study. The study was conducted in accordance with the Declaration of Helsinki (6th revision), and the protocol was approved by the Ethics Committee of Sejong General Hospital (approval number: 1810).

\section{Acknowledgment}

Not applicable.

\section{Funding}

This research received no external funding.

\section{Conflict of interest}

The authors declare no conflict of interest.

\section{Supplementary material}

Supplementary material associated with this article can be found, in the online version, at https://www.imrpre ss.com/journal/RCM/23/1/10.31083/j.rcm2301018.

\section{References}

[1] Flather MD, Yusuf S, Køber L, Pfeffer M, Hall A, Murray G, et al. Long-term ACE-inhibitor therapy in patients with heart failure or left-ventricular dysfunction: a systematic overview of data from individual patients. The Lancet. 2000; 355: 1575 1581.

[2] Pfeffer MA, McMurray JJV, Velazquez EJ, Rouleau J, Køber L, Maggioni AP, et al. Valsartan, Captopril, or both in Myocardial Infarction Complicated by Heart Failure, Left Ventricular Dysfunction, or both. New England Journal of Medicine. 2003; 349: 1893-1906.

[3] Dickstein K, Kjekshus J. Effects of losartan and captopril on mortality and morbidity in high-risk patients after acute myocardial infarction: the OPTIMAAL randomised trial. Optimal Trial in Myocardial Infarction with Angiotensin II Antagonist Losartan. Lancet. 2002; 360: 752-760.

[4] Ko D, Azizi P, Koh M, Chong A, Austin P, Stukel T, et al. Comparative effectiveness of ACE inhibitors and angiotensin receptor blockers in patients with prior myocardial infarction. Open Heart. 2019; 6: e001010.

[5] Labinjoh C, Newby DE, Pellegrini MP, Johnston NR, Boon NA, Webb DJ. Potentiation of bradykinin-induced tissue plasminogen activator release by angiotensin-converting enzyme inhibition. Journal of the American College of Cardiology. 2001; 38: $1402-1408$
[6] Byun JK, Choi BG, Rha S, Choi SY, Jeong MH. Comparison of angiotensin-converting enzyme inhibitors and angiotensin II receptor blockers in patients with diabetes mellitus and nonST-segment elevation myocardial infarction who underwent successful percutaneous coronary intervention. Atherosclerosis. 2018; 277: 130-135.

[7] Kim YH, Her A, Jeong MH, Kim B, Lee S, Hong S, et al. Comparison between Beta-Blockers with Angiotensin-Converting Enzyme Inhibitors and Beta-Blockers with Angiotensin II Type i Receptor Blockers in ST-Segment Elevation Myocardial Infarction after Successful Percutaneous Coronary Intervention with Drug-Eluting Stents. Cardiovascular Drugs and Therapy. 2019; 33: 55-67.

[8] Ponikowski P, Voors AA, Anker SD, Bueno H, Cleland JGF, Coats AJS, et al. 2016 ESC Guidelines for the diagnosis and treatment of acute and chronic heart failure: the Task Force for the diagnosis and treatment of acute and chronic heart failure of the European Society of Cardiology (ESC)Developed with the special contribution of the Heart Failure Association (HFA) of the ESC. European Heart Journal. 2016; 37: 2129-2200.

[9] Ibanez B, James S, Agewall S, Antunes MJ, Bucciarelli-Ducci C, Bueno H, et al. 2017 ESC Guidelines for the management of acute myocardial infarction in patients presenting with STsegment elevation: The Task Force for the management of acute myocardial infarction in patients presenting with ST-segment elevation of the European Society of Cardiology (ESC). European Heart Journal. 2018; 39: 119-177.

[10] O'Kane KP, Webb DJ, Collier JG, Vallance PJ. Local LNG-monomethyl-arginine attenuates the vasodilator action of bradykinin in the human forearm. British Journal of Clinical Pharmacology. 1994; 38: 311-315.

[11] Mombouli JV, Illiano S, Nagao T, Scott-Burden T, Vanhoutte PM. Potentiation of endothelium-dependent relaxations to bradykinin by angiotensin i converting enzyme inhibitors in canine coronary artery involves both endothelium-derived relaxing and hyperpolarizing factors. Circulation Research. 1992; 71: 137-144

[12] Yusuf S, Sleight P, Pogue J, Bosch J, Davies R, Dagenais G. Effects of an angiotensin-converting-enzyme inhibitor, ramipril, on cardiovascular events in high-risk patients. The New England Journal of Medicine. 2000; 342: 145-153.

[13] Ferrari R, Fox K. Insight into the mode of action of ACE inhibition in coronary artery disease: the ultimate 'EUROPA' story. Drugs. 2009; 69: 265-277.

[14] Strauss MH, Hall AS. Angiotensin receptor blockers may increase risk of myocardial infarction: unraveling the ARB-MI paradox. Circulation. 2006; 114: 838-854.

[15] Kim MP, Zhou M, Wahl LM. Angiotensin II increases human monocyte matrix metalloproteinase- 1 through the at 2 receptor and prostaglandin E2: implications for atherosclerotic plaque rupture. Journal of Leukocyte Biology. 2005; 78: 195-201.

[16] Yusuf S, Pfeffer MA, Swedberg K, Granger CB, Held P, McMurray JJV, et al. Effects of candesartan in patients with chronic heart failure and preserved left-ventricular ejection fraction: the CHARM-Preserved Trial. Lancet. 2003; 362: 777-781.

[17] Massie BM, Carson PE, McMurray JJ, Komajda M, McKelvie R, Zile MR, et al. Irbesartan in patients with heart failure and preserved ejection fraction. The New England Journal of Medicine. 2008; 359: 2456-2467.

[18] Ferrari R, Boersma E. The impact of ACE inhibition on all-cause and cardiovascular mortality in contemporary hypertension trials: a review. Expert Review of Cardiovascular Therapy. 2013; 11: 705-717.

[19] Ferrari R. RAAS inhibition and mortality in hypertension. Global Cardiology Science \& Practice. 2013; 2013: 269-278.

[20] Jung C, Elsässer A. Update ESC Guideline 2017 - Acute My- 
ocardial Infarction (STEMI). Deutsche Medizinische Wochenschrift. 2018; 143: 797-801.

[21] O'Gara PT, Kushner FG, Ascheim DD, Casey DE Jr, Chung MK, de Lemos JA, et al. 2013 ACCF/AHA guideline for the management of ST-elevation myocardial infarction: a report of the American College of Cardiology Foundation/American Heart Association Task Force on Practice Guidelines. Circulation. 2013; 127: e362-e425.

[22] Maggioni AP, Anker SD, Dahlström U, Filippatos G, Ponikowski P, Zannad F, et al. Are hospitalized or ambulatory patients with heart failure treated in accordance with European Society of Cardiology guidelines? Evidence from 12,440 patients of the ESC Heart Failure Long-Term Registry. European Journal of Heart Failure. 2013; 15: 1173-1184.

[23] Bangalore S, Makani H, Radford M, Thakur K, Toklu B, Katz $\mathrm{SD}$, et al. Clinical outcomes with beta-blockers for myocardial infarction: a meta-analysis of randomized trials. The American Journal of Medicine. 2014; 127: 939-953.

[24] Dondo TB, Hall M, West RM, Jernberg T, Lindahl B, Bueno H, et al. B-Blockers and Mortality after Acute Myocardial Infarction in Patients without Heart Failure or Ventricular Dysfunction. Journal of the American College of Cardiology. 2017; 69: 2710-2720.

[25] Khan MS, Fonarow GC, Ahmed A, Greene SJ, Vaduganathan $\mathrm{M}$, Khan $\mathrm{H}$, et al. Dose of Angiotensin-Converting Enzyme Inhibitors and Angiotensin Receptor Blockers and Outcomes in Heart Failure: a Meta-Analysis. Circulation. Heart Failure. 2017; 10: e003956.

[26] Park K, Kim Y, Kim K, Lee S, Park T, Lee S, et al. The impact of a dose of the angiotensin receptor blocker valsartan on post-myocardial infarction ventricular remodelling. ESC Heart Failure. 2018; 5: 354-363. 